\title{
Comparison of Readout-Segmented Echo-Planar Imaging and Single-Shot TSE DWI for Cholesteatoma Diagnostics
}

\author{
(D) M. Wiesmueller, (D)W. Wuest, (D) M.S. May, (D) S. Ellmann, (D) R. Heiss, (D) M. Saake, (D) R. Janka, (D) M. Uder, and (D) F.B. Laun
}

\begin{abstract}
BACKGROUND AND PURPOSE: The high diagnostic value of DWI for cholesteatoma diagnostics is undisputed. This study compares the diagnostic value of readout-segmented echo-planar DWI and single-shot TSE DWI for cholesteatoma diagnostics.

MATERIALS AND METHODS: Thirty patients with newly suspected cholesteatoma were examined with a dedicated protocol, including readout-segmented echo-planar DWI and single-shot TSE DWI at 1.5T. Acquisition parameters of both diffusion-weighted sequences were as follows: $b=1000 \mathrm{~s} / \mathrm{mm},{ }^{2}$ axial and coronal section orientations, and section thickness of $3 \mathrm{~mm}$. Image quality was evaluated by 2 readers on a 5-point Likert scale with respect to lesion conspicuity, the presence of susceptibility artifacts mimicking cholesteatomas, and overall subjective image quality. Sensitivity and specificity were calculated using histology results as the gold standard.

RESULTS: Twenty-five cases of histologically confirmed cholesteatomas were included in the study group. Lesion conspicuity was higher and fewer artifacts were found when using TSE DWI (both $P<.001$ ). The overall subjective image quality, however, was better with readout-segmented DWI. For TSE DWI, the sensitivity for readers 1 and 2 was $92 \%$ (95\% Cl, 74\%-99\%) and 88\% (95\% Cl, 69\%-97\%), respectively, while the specificity for both readers was $80 \%$ ( $95 \% \mathrm{Cl}, 28 \%-99 \%)$. For readout-segmented DWI, the sensitivity for readers 1 and 2 was $76 \%(95 \% \mathrm{Cl}, 55 \%-91 \%)$ and $68 \%(95 \% \mathrm{Cl}, 46 \%-85 \%)$, while the specificity for both readers was $60 \%(95 \% \mathrm{Cl}, 15 \%-95 \%)$.
\end{abstract}

CONCLUSIONS: The use of TSE DWI is advisable for cholesteatoma diagnostics and preferable over readout-segmented DWI.

ABBREVIATIONS: rsDWI = readout-segmented echo-planar DWI; tseDWI $=$ TSE DWI

holesteatoma is a common non-neoplastic disease in otology,

characterized by collections of trapped keratinous debris within a sack of stratified epithelium, typically found in the middle ear and capable of causing a progressive inflammatory process. ${ }^{1}$ Clinical complications include the destruction of adjacent bone and ossicular structures, which can lead to conductive or sensoneuronal hearing loss. ${ }^{2,3}$ Cholesteatoma is commonly treated with

Received October 10, 2020; accepted after revision January 23, 2021.

From the Institute of Radiology (M.W., W.W., M.S.M., S.E., R.H., M.S., R.J., M.U., F.B.L.) and Image Science Institute (M.W., W.W., M.S.M., R.H., M.S., R.J., M.U.), University Hospital Erlangen, Friedrich-Alexander-Universität Erlangen-Nürnberg, Erlangen, Germany.

Paper previously presented, in part, at: Annual Meeting of the Radiological Society of North America, November 29 to December 5, 2020; Virtual.

The position of the F.B. Laun was funded by the Deutsche Forschungsgemeinschaft (LA 2804/6-1 and LA 2804/12-1).

Please address correspondence to Marco Wiesmueller, MD, Institute of Radiology, University Hospital Erlangen, Friedrich-Alexander-University ErlangenNuremberg (FAU), Maximiliansplatz 3, 91054 Erlangen, Germany; e-mail:

marco.wiesmueller@uk-erlangen.de

- Indicates open access to non-subscribers at www.ajnr.org

http://dx.doi.org/10.3174/ajnr.A7112 surgery, ranging from focal excision to radical mastoidectomy. ${ }^{4} \mathrm{~A}$ second-look surgery procedure is typically performed within the first 2 years after the initial surgery to identify residual or recurrent cholesteatoma foci. Unlike canal wall down mastoidectomy, visual inspection of canal wall up mastoidectomy can be challenging; hence, a reliable diagnostic imaging tool is desirable for accurate follow-up diagnosis and treatment. ${ }^{5-7}$ Preoperative high-resolution CT is the method of choice for the detection of osseous disintegration and is sufficient for diagnosis; however, for recurrent cholesteatoma after surgery, its role may be more limited. ${ }^{8}$ MR imaging is suitable for the assessment pre- and postsurgery using DWI and delayed postcontrast T1-weighted spin-echo imaging, which enable differentiation between keratinous debris and noncholesteatoma findings such as granulation tissue or scar. ${ }^{9}$

The value of DWI in cholesteatoma diagnostics was initially shown using echo-planar DWI sequences. ${ }^{10-12}$ Alternative approaches have been proposed for cholesteatoma diagnostics such as diffusion-sensitized driven-equilibrium $\mathrm{DWI}^{13}$ and PROPELLER TSE DWI (tseDWI). ${ }^{14}$ Notably, tseDWI techniques introduce radiofrequency refocusing pulses between the $k$-space 
Table 1: DWI sequence parameters

\begin{tabular}{|c|c|c|}
\hline Sequence & tseDWI & rsDWI \\
\hline TR (ms) & 2000 & 4000 \\
\hline TE (ms) & 103 & $\begin{array}{c}66 \text { (and } 91 \text { for } \\
\text { phase-correction scan) }\end{array}$ \\
\hline Voxel size $\left(\mathrm{mm}^{3}\right)$ & $1.1 \times 1.5 \times 3$ & $1.4 \times 1.4 \times 3$ \\
\hline $\mathrm{FOV}\left(\mathrm{mm}^{2}\right)$ & 220 & 230 \\
\hline FOV in-phase direction & $100 \%$ & $65 \%$ \\
\hline Phase direction & $\begin{array}{l}\text { Anterior-posterior (axial), } \\
\text { right to center (coronal) }\end{array}$ & $\begin{array}{l}\text { Right to center } \\
\text { (axial and coronal) }\end{array}$ \\
\hline Phase resolution & $75 \%$ & $100 \%$ \\
\hline Partial Fourier & $50 \%$ (phase) & $87.5 \%$ (readout) \\
\hline Matrix & $192 \times 144$ & $160 \times 104$ \\
\hline Section distance & $10 \%$ & $10 \%$ \\
\hline \multirow[t]{2}{*}{ No. of slices } & 13 (axial) & 13 (axial) \\
\hline & 11 (coronal) & 11 (coronal) \\
\hline Parallel imaging & GRAPPA $\times 2$ & GRAPPA $\times 2$ \\
\hline Bandwidth (Hz/pixel) & 554 & 977 \\
\hline Echo spacing (ms) & 4.48 & 0.36 \\
\hline Readout segments & 1 & 5 \\
\hline Flip angle & $150^{\circ}$ & $180^{\circ}$ \\
\hline b-values $\left(\mathrm{s} / \mathrm{mm}^{2}\right)$ & 1000 & 0,1000 \\
\hline Averages & 10 & $\begin{array}{l}1\left(\text { for } b=0 \mathrm{~s} / \mathrm{mm}^{2}\right), \\
2\left(\text { for } b=1000 \mathrm{~s} / \mathrm{mm}^{2}\right)\end{array}$ \\
\hline Diffusion mode & 3D diagonal & 4-scan trace \\
\hline Diffusion scheme & Bipolar & Bipolar \\
\hline Acquisition time (minute:second) & 4:22 (axial), 3:42 (coronal) & $3: 06$ \\
\hline
\end{tabular}

Note:-GRAPPA indicates generalized autocalibrating partially parallel acquisition.
Patients with contraindications for MR imaging (such as a pacemaker, metal fragments, unsuitable implants, or claustrophobia) were excluded. All patients underwent an surgery after their respective MR imaging examinations, and histologic findings served as the gold standard for the presence of a cholesteatoma. Institutional review board approval was obtained, and all patients gave written informed consent.

\section{Image Acquisition}

All MR imaging examinations were performed on a $1.5 \mathrm{~T}$ MR imaging scanner (Magnetom Aera; Siemens) with a dedicated 20-channel head and neck coil using a routine examination protocol for cholesteatoma and additional rsDWI sequences as stated below.

Our routine examination protocol for cholesteatoma of the temporal region consists of a T1-weighted sequence in axial-section orientation with a $2-\mathrm{mm}$ section thickness; a T2-weighted CISS sequence in axial-section orientation with an isotropic $0.8-\mathrm{mm}$ voxel size; and a lines and are, therefore, not able to easily fulfill the Carr-PurcellMeibom-Gill Sequence condition if diffusion encoding is applied-an issue that must be addressed in the sequence design and essentially often degrades the image quality. ${ }^{15}$ Nonetheless, several studies have suggested single-shot tseDWI to be superior in terms of diagnostic accuracy compared with single-shot echo-planar DWI. ${ }^{16-18}$ The image quality of EPI in the temporal region is often degraded due to the inhomogeneous magnetic environment at the skull base. Moreover, regions adjacent to bone- or air-filled spaces can artificially appear hyperintense, which can be misleading and result in false-positive findings.

Readout-segmented echo-planar DWI (rsDWI) ${ }^{19}$ as a derivative of conventional echo-planar DWI can be used to minimize the geometric distortions to improve both image quality and diagnostic accuracy. ${ }^{20,21}$ In a recently published work by Algin et $\mathrm{al}^{21}{ }^{21}$ rsDWI proved to be superior to single-shot EPI sequences for cholesteatoma diagnostics. Hence, this study sought to compare rsDWI and tseDWI, focusing on image quality and performance in cholesteatoma diagnostics.

\section{MATERIALS AND METHODS \\ Patient Population and Study Procedure}

On the basis of the findings of a clinical assessment performed by a consultant physician for otorhinolaryngology, only patients with a newly suspected cholesteatoma were prospectively included into this study. In total, 30 patients underwent MR imaging to detect cholesteatoma and to evaluate its extension. postcontrast T1-weighted sequence with spectral fat saturation in axial- and coronal-section orientations, each with a 2 -mm section thickness. The complete neurocranium was imaged with a T2weighted FLAIR sequence in an axial-section orientation with a 5-mm section thickness.

A HASTE DWI sequence (tseDWI) and a rsDWI (readout segmentation of long variable echo trains ${ }^{19}$ ) sequence of the temporal region were measured in axial- and coronal-section orientations (see Table 1 for detailed sequence parameters). The tseDWI sequence is part of our institution's routine cholesteatoma protocol. The rsDWI sequence was added at the end of the protocol. The vendor-provided prescan normalize option was used for both DWI sequences to correct for spatially varying coil-sensitivity profiles and to ensure homogeneous image signal intensity. The tseDWI was interpolated to a doubled matrix size using the vendor-provided standard settings.

\section{Image Analysis}

The image analysis was independently performed by 2 experienced radiologists (reader 1 with 6 years of experience and reader 2 with 9 years of experience in head and neck MR imaging, respectively) who were blinded to information on patient status and additional imaging data. First, each reader evaluated only tseDWI data in a random order. Then, a few days later, rsDWI data were assessed in a different order to avoid recall bias. In the readers' opinions, if both ears were affected, further documentation referred to only the left ear. The histopathologic reference was unknown at the time of assessment.

Both readers rated the image quality for each of the 4 diffusion-weighted sequences (ie, tseDWI axial, tseDWI coronal, 

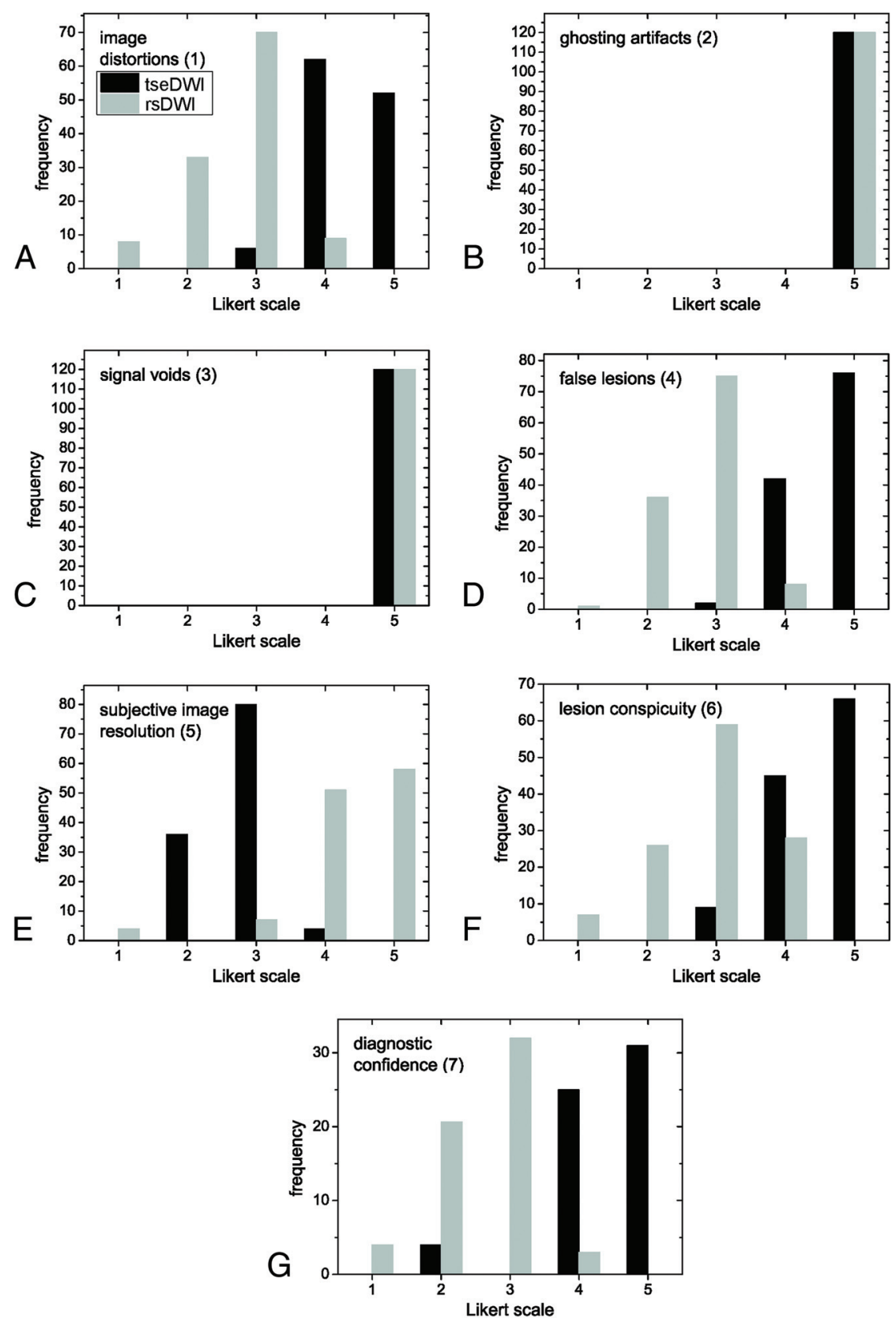

FIG 1. Histograms displaying Likert scale scores ranging from 1 (worst) to 5 (best) points. The histograms show the combined frequencies of both readers. The frequencies of axial and coronal images were added.

rsDWI axial, and rsDWI coronal) using the following categories on a 5-point Likert scale:

1) Prominence of geometric image distortions in the relevant temporal region, ie, inner ear, middle ear, and outer auditory canal ( $1=$ very strong, $2=$ strong, $3=$ medium, $4=$ small, and $5=$ negligible).

2) Prominence of ghosting artifacts in the relevant temporal region $(1=$ very strong, $2=$ strong, $3=$ medium, $4=$ small, and $5=$ negligible).

3) Prominence of signal voids or other artifacts in the relevant temporal region $(1=$ very strong, $2=$ strong, $3=$ medium, $4=$ small, and $5=$ negligible)—notably, signal voids might originate from pulsatile motion.

4) Presence of bright-appearing regions that might be mistaken for a true lesion $(1=$ present and not distinguishable from a true lesion, $2=$ present and hardly distinguishable from the true lesion, 3 = present but clearly distinguishable, $4=$ hardly present, and $5=$ not present).

5) Subjective rating of image resolution $(1=$ very low, $2=$ low, $3=$ medium, $4=$ good, and $5=$ very good).

Additionally, the readers evaluated the diagnostic properties of the datasets (ie, lesion conspicuity) as follows, also using a 5-point Likert scale:

6) Lesion conspicuity ( $1=$ very bad, $2=$ bad, $3=$ medium, $4=$ good, $5=$ very good). Each reader provided 1 rating each for the axial and coronal datasets, respectively.

Then, the readers rated the 2 tseDWI datasets (axial and coronal combined) and the 2 rsDWI datasets (axial and coronal combined):

7) Diagnostic confidence ( $1=$ very low, $2=$ low, $3=$ medium, $4=$ high, and $5=$ very high).

8) Each reader had to decide whether a cholesteatoma was present or not (yes/ no). This decision was made on the basis of the signal intensity in a supposed lesion relative to that of the adjacent brain tissue. The decision was "yes" if a hyperintense signal could be detected and "no" otherwise.

Subsequently, in a second independent assessment cycle, tseDWI and rsDWI data were simultaneously compared. The readers rated the 2 tseDWI datasets (axial and coronal combined) versus the 2 rsDWI datasets (axial and coronal combined) regarding the following:

9) Lesion conspicuity when comparing tseDWI versus rsDWI ( 2 = much better with tseDWI, $1=$ better with tseDWI, $0=$ equal, $-1=$ better with rsDWI, and $-2=$ much better with rsDWI).

10) Subjective diagnostic confidence $(2=$ much better with tseDWI, $1=$ better with tseDWI, $0=$ equal, $-1=$ better with rsDWI, and $-2=$ much better with rsDWI).

\section{Statistics}

Statistical analysis was performed individually for each reader and for each image dataset, calculating (separately for the 2 


\begin{tabular}{|c|c|c|c|c|c|c|c|}
\hline \multirow[b]{2}{*}{ Category } & \multirow[b]{2}{*}{ Coronal/Axial } & \multicolumn{2}{|c|}{$\begin{array}{l}\text { tseDWI (Likert Categories } \\
4 \text { and } 5 \text { ) }\end{array}$} & \multicolumn{2}{|c|}{$\begin{array}{c}\text { rsDWI (Likert Categories } \\
4 \text { and } 5 \text { ) } \\
\end{array}$} & \multicolumn{2}{|c|}{$\begin{array}{c}\text { Mean Difference } \\
\text { (tseDWI and rsDWI) }\end{array}$} \\
\hline & & Reader 1 & Reader 2 & Reader 1 & Reader 2 & Reader 1 & Reader 2 \\
\hline 1) Geometric image distortion & Cor & $97 \%$ & $97 \%$ & $0 \%$ & $0 \%$ & 1.9 & 2 \\
\hline 1) Geometric image distortion & $A x$ & $93 \%$ & $93 \%$ & $17 \%$ & $13 \%$ & 1.5 & 1.5 \\
\hline 2) Ghosting artifacts & Cor & $100 \%$ & $100 \%$ & $100 \%$ & $100 \%$ & 0 & 0 \\
\hline 2) Ghosting artifacts & Ax & $100 \%$ & $100 \%$ & $100 \%$ & $100 \%$ & 0 & 0 \\
\hline 3) Signal voids or other artifacts & Cor & $100 \%$ & $100 \%$ & $100 \%$ & $100 \%$ & 0 & 0 \\
\hline 3) Signal voids or other artifacts & $A x$ & $100 \%$ & $100 \%$ & $100 \%$ & $100 \%$ & 0 & 0 \\
\hline 4) Bright-appearing region & Cor & $97 \%$ & $97 \%$ & $0 \%$ & $0 \%$ & 2 & 2 \\
\hline 4) Bright-appearing region & $A x$ & $100 \%$ & $100 \%$ & $13 \%$ & $13 \%$ & 1.7 & 1.8 \\
\hline 5) Subjective image resolution & Cor & $3 \%$ & $3 \%$ & $90 \%$ & $90 \%$ & -1.6 & -1.7 \\
\hline 5) Subjective image resolution & Ax & $3 \%$ & $3 \%$ & $90 \%$ & $93 \%$ & -1.5 & -1.6 \\
\hline 6) Lesion conspicuity & Cor & $97 \%$ & $93 \%$ & $33 \%$ & $23 \%$ & 1.6 & 1.7 \\
\hline 6) Lesion conspicuity & $\mathrm{Ax}$ & $90 \%$ & $90 \%$ & $20 \%$ & $17 \%$ & 1.5 & 1.5 \\
\hline 7) Diagnostic confidence & Cor/Ax & $93 \%$ & $93 \%$ & $7 \%$ & $3 \%$ & 1.8 & 1.8 \\
\hline
\end{tabular}

Note:-Cor indicates coronal; Ax, axial.

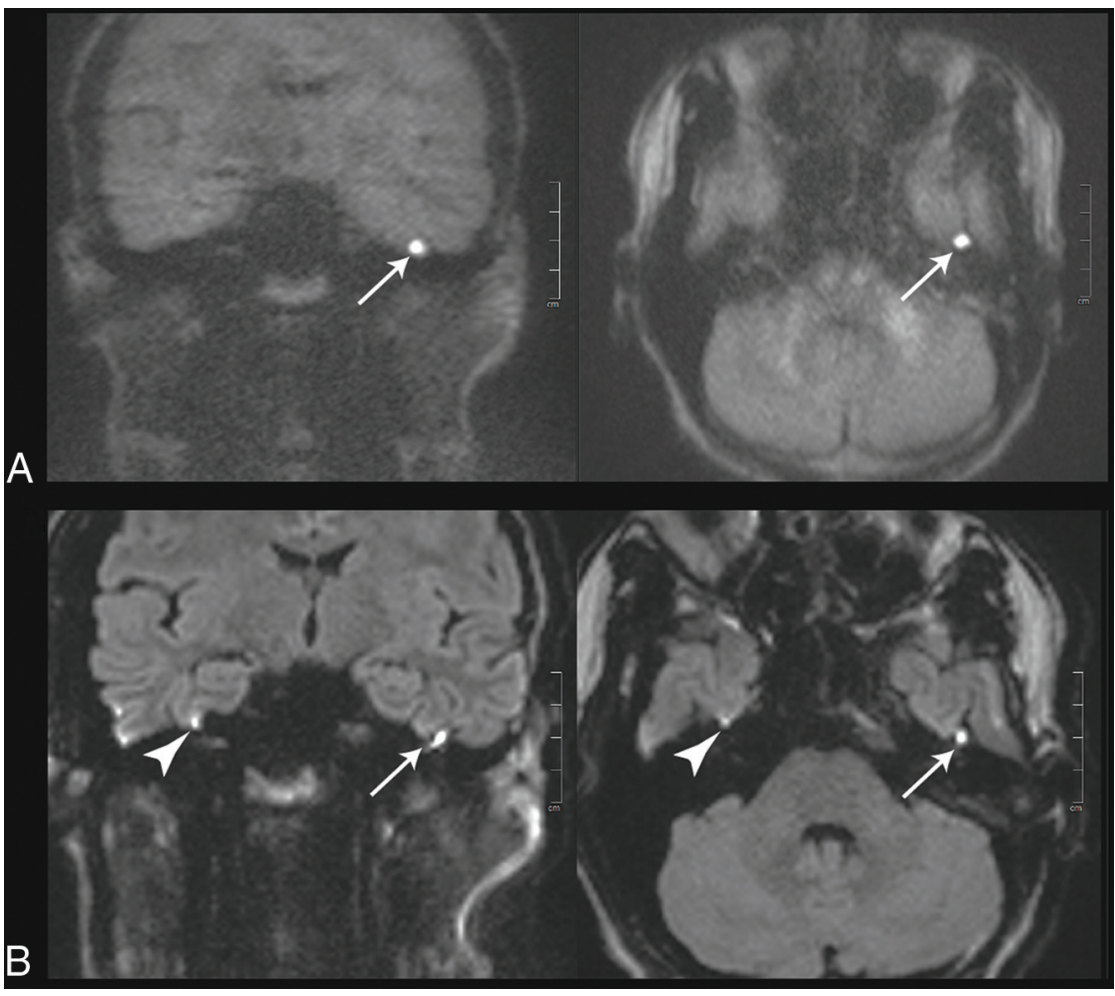

FIG 2. Images of a 20-year-old patient with a left-sided cholesteatoma (white arrows). A, tseDWI. $B$, rsDWI. The lesion can be seen clearly in tseDWI and rsDWI. It is, however, not spheric in the coronal rsDWI but rather is elongated and tilted due to image distortions in phase directions. Field inhomogeneities generate a bright spot in the rsDWI that might be mistaken for a lesion (arrowheads). The tseDWI displayed in A is more blurred than the rsDWI and shows less contrast in the brain. For example, unlike in the rsDWl, both white matter and gray matter are not discernible in the tseDWl.

groups of patients with histologically proved cholesteatomas and no cholesteatomas, respectively) the sensitivity and specificity with 95\% confidence intervals using the ClopperPearson method. Furthermore, the McNemar test was performed to compare the $2 \mathrm{MR}$ imaging sequences per these measures of diagnostic accuracy, while interrater agreement was evaluated using the Cohen $\kappa$ value; in this context, $\kappa$ was interpreted as follows: $0<\kappa \leq 0.2$ indicated slight agreement, $0.2<\kappa \leq 0.4$ indicated fair agreement, $0.4<\kappa \leq 0.6$ indicated moderate agreement, $0.6<\kappa \leq 0.8$ indicated substantial agreement, 0.8 $<\kappa \leq 1.0$ indicated almost perfect agreement, and $\kappa=1$ indicated perfect agreement. A comparison between tseDWI and rsDWI ratings was performed using a nonparametric Wilcoxon rank sum test. Significance was accepted for $P$ values $<.05$. Statistical analysis was performed using SPSS Statistics, Version 24 (IBM) and R statistical and computing software, Version 3.5.3 (http://www.r-project.org/).

\section{RESULTS}

\section{Patient Population}

The study population consisted of 13 female and 17 male patients with a mean age of 46 (SD, 16) years (range, 1879 years). Twenty-five of the 30 included patients $(83 \%)$ were diagnosed with cholesteatoma after surgical excision and histopathologic confirmation, with 22 of 25 patients $(88 \%)$ with unilateral cholesteatoma and 3 patients with bilateral cholesteatoma (12\%). The mean lesion diameter was $0.6(\mathrm{SD}, 0.4) \mathrm{cm}$ (median, $0.5 \mathrm{~cm}$; range, $0.1-1.6 \mathrm{~cm}$ ).

\section{Diagnostic Performance}

Regarding the 25 patients with cholesteatoma, reader 1 found 23 cholesteatomas with tseDWI and 19 with rsDWI, with 18 being 


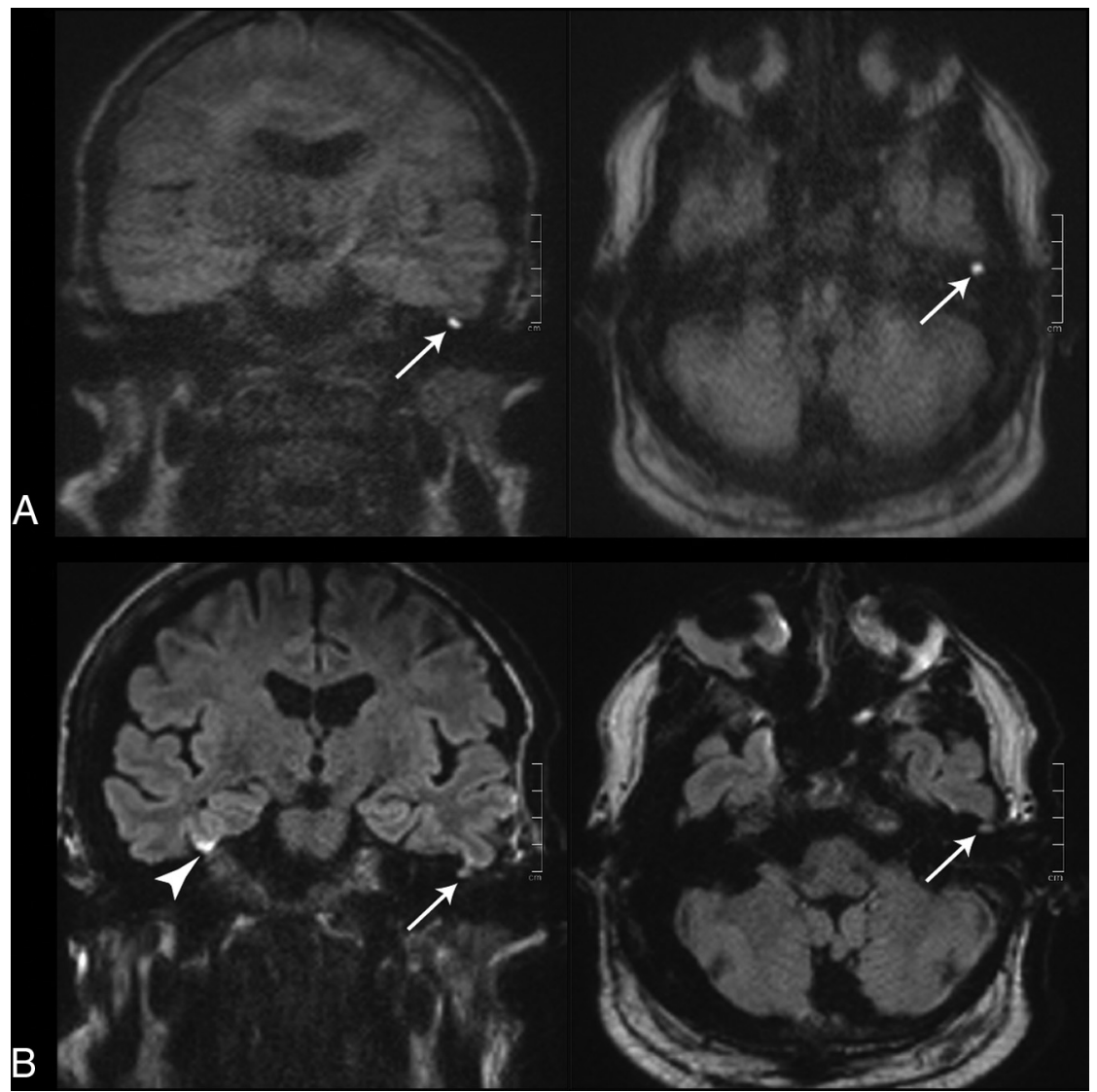

FIG 3. Images of a 55-year-old patient with a left-sided cholesteatoma (white arrows). A, tseDWI. $B$, rsDWI. The lesion can be seen clearly in the tseDWI. In the rsDWI, however, the lesion is displayed with reduced contrast and is hardly visible; therefore, both readers did not diagnose a cholesteatoma in this case in the rsDWI dataset. Again, a bright spot is present in the coronal rsDWI next to the temporal bone on the right side due to field inhomogeneities, which might be mistaken for a true lesion (arrowhead).

concordantly found with both sequences. The sensitivity for tseDWI and rsDWI in this case was 92\% (95\% CI, 74\%-99\%) and $76 \%$ (95\% CI, 55\%-91\%) (McNemar $P=.22$ ). Reader 2 correctly found 22 cholesteatomas with tseDWI and 17 with rsDWI, with 16 being concordantly found with both sequences. The sensitivity for tseDWI and rsDWI was 88\% (95\% CI, 69\%-97\%) and 68\% (95\% CI, 46\%-85\%) (McNemar $P=.13$ ).

Regarding the 5 patients without cholesteatomas, reader 1 negatively diagnosed 4 patients by tseDWI; 3 patients were ultimately also classified correctly by rsDWI, leading to specificities of $80 \%$ (95\% CI, 28\%-99\%) and 60\% (95\% CI, 15\%-95\%), respectively (McNemar $P=1$ ). Reader 2 classified 2 patients concordantly correctly negative with both sequences. Meanwhile, 2 patients were correctly classified with tseDWI but not with rsDWI, and 1 patient was correctly classified by rsDWI but not by tseDWI. Thus, the specificity for tseDWI was $80 \%$ (95\% CI, $28 \%-99 \%)$, and for rsDWI, it was $60 \%$ (95\% CI, $15 \%-95 \%)$ $(\mathrm{McNemar} P=1)$.

The overall agreement between both readers concerning the presence of a cholesteatoma was $97 \%(\kappa=0.9)$ for tseDWI and $87 \%(\kappa=0.7)$ for rsDWI, respectively.

\section{Likert Score Ratings}

Frequencies of Likert scores per category are summarized in Fig 1 and Table 2. Relevant ghosting artifacts and signal voids were not observed by the readers for both rsDWI and tseDWI. rsDWI outperformed tseDWI only in terms of the subjective image resolution, while tseDWI performed much better in terms of handling image distortions and lesion conspicuity. For rsDWI, the readers more frequently observed the presence of bright-appearing regions outside the lesion that might be mistaken for a true lesion. The diagnostic confidence was higher for tseDWI. Representative images are provided in Figs 2-4.

Figure 5 shows the comparative reader evaluations performed with possible Likert scores ranging from -2 to 2 points (categories 9 and 10). Notably, tseDWI always performed equal to or even better than rsDWI; the readers assigned better lesion conspicuity in $91 \%$ of cases and better diagnostic confidence in 95\% of cases for tseDWI, respectively.

The differences between tseDWI and rsDWI were significant $(P<.001)$ in all cases, ie, for all section orientations and both readers, except with regard to signal voids and ghosting artifacts $(P=1)$. Table 3 summarizes the $\kappa$ values. A moderateto-perfect degree of interreader agreement was observed for all evaluations.

\section{DISCUSSION}

In this study, we found that single-shot tseDWI outperformed rsDWI with respect to diagnostic performance, lesion conspicuity, the presence of false-positive findings, and subjective diagnostic confidence for imaging cholesteatomas. tseDWI showed greater sensitivity and specificity compared with rsDWI, albeit without verifiable statistical significance. Notably, the better subjective image resolution of rsDWI did not translate into a generally improved level of performance in comparison with tseDWI. So far, to our knowledge, only a few studies have evaluated the impact of rsDWI in cholesteatoma diagnostics.

The reductions in susceptibility artifacts that we observed with tseDWI in comparison with rsDWI are in line with the technical considerations that can be made for the 2 sequences. For multishot EPI, the susceptibility-induced distortion is $\Delta r_{\mathrm{EPI}}=\frac{\mathrm{t}_{\text {line }} \cdot \Delta \nu}{N_{\text {shot }}} \times \mathrm{FoV}_{\text {phase }}$, with the acquisition duration of $1 k$ space line reflected as $t_{\text {line, }}$, the susceptibility-induced off- 


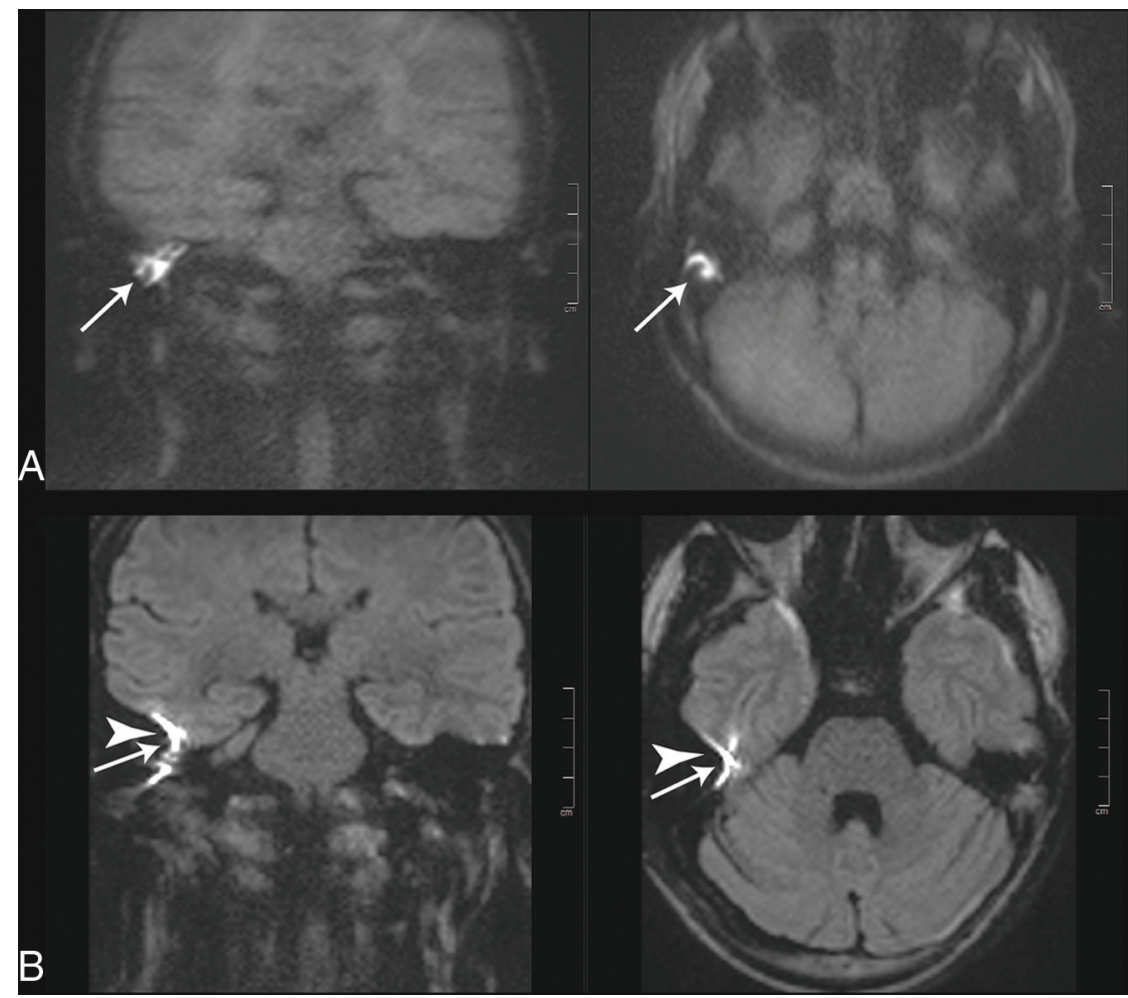

FIG 4. Images of a 38-year-old patient with a recurrent cholesteatoma (white arrows). A stapes prosthesis implant caused major image distortions in the rsDWI (arrowheads).
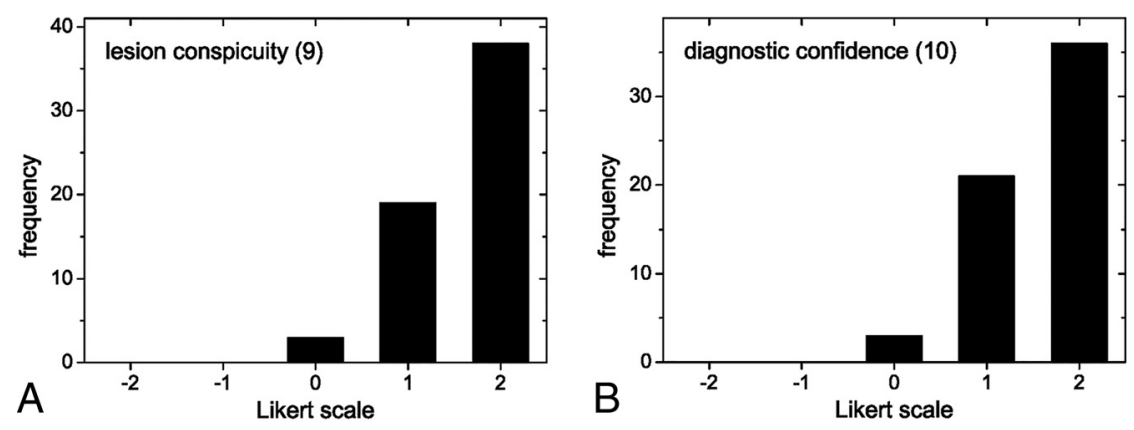

FIG 5. Histograms displaying the scores obtained with Likert scores ranging from -2 (rsDWI much better than tseDWI) to 2 points (tseDWI much better than rsDWI). The histograms show the added frequencies of both readers.

resonance stated as $\Delta \nu$, and the number of shots represented as $N_{\text {shot. }}{ }^{22}$ This relation highlights the advantage of multishot EPI techniques in comparison with single-shot EPI, for which $N_{\text {shot }}=1$. Meanwhile, the susceptibility-induced distortion in tseDWI is $\Delta r_{\text {tseDWI }}=\frac{t_{\text {line }} \cdot \Delta \nu}{N_{\text {voxel, }} \text { read }} \times \mathrm{FoV}_{\text {read }}$, with the number of voxels in the read direction being $N_{\text {voxel, read. }}{ }^{23}$ Because $N_{\text {voxel, read }}$ is less than $N_{\text {shot }}$ in our setting, the image distortions for tseDWI are technically expected to be much smaller than those for rsDWI. We used a longer TE value for tseDWI (103 ms), which might explain the better visibility of lesions assessed with tseDWI than with rsDWI. This setting for rsDWI was chosen using the vendor-provided standard option for rsDWI, which minimizes the TE and is not variable. This contrast issue might be addressed by prolonging the TE for rsDWI, but this would not solve the problem of false-positive hyperintense susceptibility artifacts due to adjacent bone and air interfaces in the temporal region in rsDWI. These are caused by field inhomogeneities and do not depend on TE. ${ }^{22}$

We could not confirm the high sensitivity and specificity values for rsDWI reported by both Fischer et $\mathrm{al}^{24}$ and Algin et al. ${ }^{21}$ In contrast to in our study, Algin et al used longer TEs for rsDWI (91-124 versus $66 \mathrm{~ms}$ ). This might increase the T2 hyperintense signal of cholesteatomas, which could possibly lead to a higher detection rate. ${ }^{25}$ Another difference that might explain a higher detection rate is the greater size of cholesteatomas in their study group (median, 0.8 versus $0.5 \mathrm{~cm}$ ). Because we were not able to track and compare all sequence parameters and lesion sizes of Fischer et al and Algin et al, we found it difficult to make statements on the origin of the observed differences.

Dudau et $\mathrm{al}^{26}$ recently conducted a similar study comparing tseDWI and rsDWI, and several of their findings are in line with ours. In particular, these authors reported a good overall agreement between the 2 sequences with similar levels of diagnostic performance for rsDWI and tseDWI (positive predictive value $=93 \%$ versus $92.5 \%$, negative predictive value $=70 \%$ versus $80 \%$ ), while we observed a drop in the diagnostic performance for the rsDWI sequence. Furthermore, concerning discrepancies between tseDWI and rsDWI, they found a greater proportion of false-negative results for rsDWI, which is in line with our results. Many of the settings that Dudau et al used are quite similar to ours (eg, same scanner, sequence, similar TEs). They used a smaller voxel size for rsDWI acquisitions than we did $\left(1.2 \times 1.2 \times 2 \mathrm{~mm}^{3}\right.$ versus $\left.1.4 \times 1.4 \times 3 \mathrm{~mm}^{3}\right)$. This may have been beneficial during imaging because it reduces partial volume effects and thus can make small lesions appear brighter. However, they did not report the lesion sizes, which might be different from those in our study. Moreover, their main detection criterion was also the presence of signal hyperintensity, but perhaps, they also used a better reading strategy, potentially classifying smaller hyperintensities as lesions. On the one hand, this could indicate that an improvement of our reading strategy might be possible, while on the other, it might also indicate that the diagnostic performance of 


\begin{tabular}{|c|c|c|c|c|}
\hline & $\kappa$ (tseDWI, Axial) & $\begin{array}{c}\kappa \text { (tseDWI, } \\
\text { Coronal) }\end{array}$ & $\begin{array}{c}\text { к (rsDWI, } \\
\text { Axial) }\end{array}$ & $\begin{array}{c}\kappa \text { (rsDWI, } \\
\text { Coronal) }\end{array}$ \\
\hline 1) Geometric image distortion & 0.64 & 0.62 & 0.83 & 0.58 \\
\hline 2) Ghosting artifacts & 1 & 1 & 1 & 1 \\
\hline 3) Signal voids & 1 & 1 & 1 & 1 \\
\hline 4) Bright-appearing region & 0.79 & 0.78 & 0.82 & 0.78 \\
\hline 5) Subjective image resolution & 0.79 & 0.78 & 0.71 & 0.77 \\
\hline 6) Lesion conspicuity & 0.71 & 0.62 & 0.83 & 0.53 \\
\hline \multirow[t]{2}{*}{$\begin{array}{l}\text { 7) Diagnostic confidence with } \kappa \text { (tseDWI, axial and coronal combined) } \\
\text { and } \kappa(\mathrm{rsDWI} \text {, axial and coronal combined) }\end{array}$} & $\begin{array}{c}\kappa(\text { tseDWI) } \\
0.82\end{array}$ & $\begin{array}{c}\kappa(\mathrm{rsDWI}) \\
0.77\end{array}$ & & \\
\hline & $\kappa($ tseDWI vs. rsDWI) & & & \\
\hline 9) Lesion conspicuity with $\kappa$ (tseDWI vs. rsDWI) & 0.80 & & & \\
\hline 10) Subjective diagnostic confidence & 0.68 & & & \\
\hline
\end{tabular}

tseDWI is more robust when used in different centers and that its use would thus be recommendable.

As stated above, tseDWI revealed more true-positive findings compared with rsDWI, but we also observed false-negative findings within the tseDWI datasets. In the literature, this deficiency is reported to be due to motion artifacts or empty retraction pockets. ${ }^{27,28}$ According to histopathologic results, all patients with falsenegative MR imaging results in our study had only residual portions of cholesteatomas measuring $<2 \mathrm{~mm}$. This is in line with the results published by De Foer et al, ${ }^{17}$ who proposed $2 \mathrm{~mm}$ as a size limit for accurate diagnosis when using single-shot tseDWI. Furthermore, the lack of a clear visualization of anatomic landmarks in singleshot tseDWI sequences can be considered a major limitation that could prevent the exact spatial description of a cholesteatoma in the temporal bone. ${ }^{17}$ Thus, a substantially better depiction of anatomic details would potentially help to overcome this diagnostic limitation, especially in patients with residual cholesteatoma before secondlook surgery. In 2 recently published studies, it has been shown that coregistration of DWI data with CT or T2-weighted cisternography may help in this regard. ${ }^{29,30}$ In theory, a promising approach would be rsDWI, with its better ability to depict anatomic details-if the drawbacks described in our study can be overcome.

Several possibilities exist to achieve further improvement in the performance of the rsDWI sequence, which aims to reduce the susceptibility-induced artifacts. The use of higher parallel imaging acceleration factors might become possible with the use of head coils with 64,96 , or more channels, ${ }^{31}$ which could reduce susceptibility-induced image distortions. These distortions might also be reduced by involving postprocessing schemes. ${ }^{32}$ We used a scanner with a maximal gradient strength of $40 \mathrm{mT} / \mathrm{m}$; in comparison, newer whole-body scanners can provide values of $80 \mathrm{mT} / \mathrm{m}$, while dedicated head scanners offer $300 \mathrm{mT} / \mathrm{m}$ of gradient strength. ${ }^{33}$ Using such systems might allow very high-acquisition bandwidths, which could decrease the severity of susceptibility-induced image distortions. Also, a further approach to minimize such distortions may be the use of reduced-FOV excitations. ${ }^{34,35}$

In our setting, the acquisition time of the rsDWI sequence was shorter than that of the routine tseDWI sequence. Most important, we used the vendor-provided setting for tseDWI, which involves the acquisition of 10 averages and prolongs the acquisition time more than the acquisition of multiple segments with $\mathrm{rsDWI}(n=5$ in our study).
Our study has several limitations. A quantitative evaluation of imaging features such as the signal-to-noise ratio was not performed. With multichannel coils, an evaluation of the signal-tonoise ratio would have necessitated the acquisition of $>1$ dataset for each setting, ${ }^{36}$ which contrasted with our desire to limit the total acquisition time. Furthermore, we did not run a qualityassurance program with dedicated phantoms ${ }^{37}$ during the study, and we did not evaluate computed b-value images, possibly constituting additional steps to improve the final diagnostic performance. $^{38}$

\section{CONCLUSIONS}

In cholesteatoma diagnostics, the use of single-shot tseDWI is recommended instead of echo-planar rsDWI.

Disclosures: Wolfgang Wuest-UNRELATED: Consultancy: Siemens; Payment for Lectures Including Service on Speakers Bureaus: Siemens. Matthias S. MayUNRELATED: Payment for Lectures Including Service on Speakers Bureaus: Siemens, Comments: lectures, services on speakers bureau. Rafael HeissUNRELATED: Payment for Lectures Including Service on Speakers Bureaus: Siemens. Marc Saake-UNRELATED: Payment for Lectures Including Service on Speakers Bureaus: Siemens. Rolf Janka-UNRELATED: Payment for Lectures Including Service on Speakers Bureaus: Siemens, Bracco Diagnostics. Frederik B. Laun-RELATED: Grant: Deutsche Forschungsgemeinschaft, Comments: DFG LA 2804/6-1 and LA 2804/12-1.* *Money paid to the institution.

\section{REFERENCES}

1. Swartz JD. Cholesteatomas of the middle ear: diagnosis, etiology, and complications. Radiol Clin North Am 1984;22:15-35 Medline

2. Yung M, Tono T, Olszewska E, et al. EAONO/JOS Joint Consensus Statements on the Definitions, Classification and Staging of Middle Ear Cholesteatoma. J Int Adv Otol 2017;13:1-8 CrossRef Medline

3. Suzuki C, Ohtani I. Bone destruction resulting from rupture of a cholesteatoma sac: temporal bone pathology. Otol Neurotol 2004;25:674-77 Medline

4. Prasad SC, Piras G, Piccirillo E, et al. Surgical strategy and facial nerve outcomes in petrous bone cholesteatoma. Audiol Neurootol 2016;21:275-85 CrossRef Medline

5. Foti G, Beltramello A, Minerva G, et al. Identification of residualrecurrent cholesteatoma in operated ears: diagnostic accuracy of dual-energy CT and MRI. Radiol Med 2019;124:478-86 CrossRef Medline

6. Bazzi K, Wong E, Jufas N, et al. Diffusion-weighted magnetic resonance imaging in the detection of residual and recurrent cholesteatoma in children: a systematic review and meta-analysis. Int $J$ Pediatr Otorhinolaryngol 2019;118:90-96 CrossRef Medline 
7. Gouda M, Nasr WF, Elbary ME, et al. MRI as an alternative to second look mastoid surgery. Indian J Otolaryngol Head Neck Surg 2018;70:410-14 CrossRef Medline

8. Migirov L, Tal S, Eyal A, et al. MRI, not CT, to rule out recurrent cholesteatoma and avoid unnecessary second-look mastoidectomy. Isr Med Assoc J 2009;11:144-146 Medline

9. Williams MT, Ayache D, Alberti C, et al. Detection of postoperative residual cholesteatoma with delayed contrast-enhanced MR imaging: initial findings. Eur Radiol 2003;13:169-74 CrossRef Medline

10. Maheshwari S, Mukherji SK. Diffusion-weighted imaging for differentiating recurrent cholesteatoma from granulation tissue after mastoidectomy: case report. AJNR Am J Neuroradiol 2002;23:84749 Medline

11. Aikele P, Kittner T, Offergeld C, et al. Diffusion-weighted MR imaging of cholesteatoma in pediatric and adult patients who have undergone middle ear surgery. AJR Am J Roentgenol 2003;181:26165 CrossRef Medline

12. Vercruysse JP, De Foer B, Pouillon M, et al. The value of diffusionweighted MR imaging in the diagnosis of primary acquired and residual cholesteatoma: a surgical verified study of 100 patients. Eur Radiol 2006;16:1461-67 CrossRef Medline

13. Yamashita K, Yoshiura T, Hiwatashi A, et al. High-resolution threedimensional diffusion-weighted imaging of middle ear cholesteatoma at 3.0 T MRI: usefulness of 3D turbo field-echo with diffusion-sensitized driven-equilibrium preparation (TFE-DSDE) compared to single-shot echo-planar imaging. Eur J Radiol 2013;82: e471-75 CrossRef Medline

14. Kasbekar AV, Scoffings DJ, Kenway B, et al. Non echo planar, diffusion-weighted magnetic resonance imaging (periodically rotated overlapping parallel lines with enhanced reconstruction sequence) compared with echo planar imaging for the detection of middleear cholesteatoma. J Laryngol Otol 2011;125:376-80 CrossRef Medline

15. Alsop DC. Phase insensitive preparation of single-shot RARE: application to diffusion imaging in humans. Magn Reson Med 1997;38:527-33 CrossRef Medline

16. Dubrulle F, Souillard R, Chechin D, et al. Diffusion-weighted MR imaging sequence in the detection of postoperative recurrent cholesteatoma. Radiology 2006;238:604-10 CrossRef Medline

17. De Foer B, Vercruysse JP, Bernaerts A, et al. The value of single-shot turbo spin-echo diffusion-weighted MR imaging in the detection of middle ear cholesteatoma. Neuroradiology 2007;49:841-48 CrossRef Medline

18. Pizzini FB, Barbieri F, Beltramello A, et al. HASTE diffusionweighted 3-Tesla magnetic resonance imaging in the diagnosis of primary and relapsing cholesteatoma. Otol Neurotol 2010;31:596602 CrossRef Medline

19. Porter DA, Heidemann RM. High resolution diffusion-weighted imaging using readout-segmented echo-planar imaging, parallel imaging and a two-dimensional navigator-based reacquisition. Magn Reson Med 2009;62:468-75 CrossRef Medline

20. Yamashita K, Yoshiura T, Hiwatashi A, et al. Detection of middle ear cholesteatoma by diffusion-weighted MR imaging: multishot echo-planar imaging compared with single-shot echo-planar imaging. AJNR Am J Neuroradiol 2011;32:1915-18 CrossRef Medline

21. Algin O, Aydin H, Ozmen E, et al. Detection of cholesteatoma: high-resolution DWI using RS-EPI and parallel imaging at 3 Tesla. J Neuroradiol 2017;44:388-94 CrossRef Medline
22. Jones DK. Diffusion MRI: Theory, Methods, and Applications. Oxford University Press; 2011

23. Emmerich J, Laun FB, Pfaffenberger A, et al. Technical Note: on the size of susceptibility-induced MR image distortions in prostate and cervix in the context of MR-guided radiation therapy. Med Phys 2018;45:1586-93 CrossRef Medline

24. Fischer N, Schartinger VH, Dejaco D, et al. Readout-segmented echo-planar DWI for the detection of cholesteatomas: correlation with surgical validation. AJNR Am J Neuroradiol 2019;40:1055-59 CrossRef Medline

25. Fitzek C, Mewes T, Fitzek S, et al. Diffusion-weighted MRI of cholesteatomas of the petrous bone. J Magn Reson Imaging 2002;15:636-41 CrossRef Medline

26. Dudau C, Draper A, Gkagkanasiou M, et al. Cholesteatoma: multishot echo-planar vs non echo-planar diffusion-weighted MRI for the prediction of middle ear and mastoid cholesteatoma. BJR Open 2019;1:20180015 CrossRef Medline

27. Henninger B, Kremser C. Diffusion-weighted imaging for the detection and evaluation of cholesteatoma. World $J$ Radiol 2017;9:217-22 CrossRef Medline

28. Dremmen MH, Hofman PA, Hof JR, et al. The diagnostic accuracy of non-echo-planar diffusion-weighted imaging in the detection of residual and/or recurrent cholesteatoma of the temporal bone. AJNR Am J Neuroradiol 2012;33:439-44 CrossRef Medline

29. Locketz GD, Li PM, Fischbein NJ, et al. Fusion of computed tomography and PROPELLER diffusion-weighted magnetic resonance imaging for the detection and localization of middle ear cholesteatoma. JAMA Otolaryngol Head Neck Surg 2016;142:947-53 CrossRef Medline

30. Benson JC, Carlson ML, Yin L, et al. Cholesteatoma localization using fused diffusion-weighted images and thin-slice T2 weighted images. Laryngoscope 2020 Nov 3. [Epub ahead of print] CrossRef Medline

31. Wiggins GC, Polimeni JR, Potthast A, et al. 96-Channel receive-only head coil for 3 Tesla: design optimization and evaluation. Magn Reson Med 2009;62:754-62 CrossRef Medline

32. Andersson JLR, Skare S, Ashburner J. How to correct susceptibility distortions in spin-echo echo-planar images: application to diffusion tensor imaging. Neuroimage 2003;20:870-88 CrossRef Medline

33. Setsompop K, Kimmlingen R, Eberlein E, et al. Pushing the limits of in vivo diffusion MRI for the Human Connectome Project. Neuroimage 2013;80:220-33 CrossRef Medline

34. Dowell NG, Jenkins TM, Ciccarelli O, et al. Contiguous-slice zonally oblique multislice (CO-ZOOM) diffusion tensor imaging: examples of in vivo spinal cord and optic nerve applications. J Magn Reson Imaging 2009;29:454-60 CrossRef Medline

35. Laun F, Stieltjes B, Schluter M, et al. Reproducible evaluation of spinal cord DTI using an optimized inner volume sequence in combination with probabilistic ROI analysis. Z Med Phys 2009;19:11-20 CrossRef Medline

36. Goerner FL, Clarke GD. Measuring signal-to-noise ratio in partially parallel imaging MRI. Med Phys 2011;38:5049-57 CrossRef Medline

37. Wagner F, Laun FB, Kuder TA, et al. Temperature and concentration calibration of aqueous polyvinylpyrrolidone (PVP) solutions for isotropic diffusion MRI phantoms. PLoS One 2017;12:e179276 CrossRef Medline

38. Yamashita $\mathrm{K}$, Hiwatashi A, Togao O, et al. Improved visualization of middle ear cholesteatoma with computed diffusion-weighted imaging. Magn Reson Med Sci 2019;18:233-37 CrossRef Medline 